\title{
PODEMOS, UN PARTI-MOUVEMENT
}

Manuel Cervera-Marzal

\author{
La Découverte | « Mouvements »
}

2018/2 nº 94 | pages 87 à 97

ISSN 1291-6412

ISBN 9782348036125

Article disponible en ligne à l'adresse :

https://www.cairn.info/revue-mouvements-2018-2-page-87.htm

\section{Pour citer cet article :}

Manuel Cervera-Marzal, "Podemos, un parti-mouvement », Mouvements 2018/2 ( ${ }^{\circ}$ 94), p. 87-97.

DOI 10.3917/mouv.094.0087

Distribution électronique Cairn.info pour La Découverte.

(C) La Découverte. Tous droits réservés pour tous pays.

La reproduction ou représentation de cet article, notamment par photocopie, n'est autorisée que dans les limites des conditions générales d'utilisation du site ou, le cas échéant, des conditions générales de la licence souscrite par votre établissement. Toute autre reproduction ou représentation, en tout ou partie, sous quelque forme et de quelque manière que ce soit, est interdite sauf accord préalable et écrit de l'éditeur, en dehors des cas prévus par la législation en vigueur en France. Il est précisé que son stockage dans une base de données est également interdit. 


\section{Podemos, un parti-mouvement}

Lorsqu'ils lancent leur projet en janvier 2014, les fondateur.trice.s de Podemos parlent de " mouvement politique " et promettent de ne pas créer " un parti de plus ". En s'engageant dans la compétition électorale, ils et elles sont toutefois amené.e.s à mettre en place une structure partisane. Podemos est-il pour autant un parti comme les autres ? Cet article interroge la notion de " parti mouvement ", en montrant comment Podemos se distingue des partis traditionnels et quels sont les clivages en interne.
$\mathrm{L}$ e 12 janvier 2014, le site d'information Público.es publie un manifeste intitulé "Avancer ses pions: convertir l'indignation en changement politique ". Le texte est signé par une trentaine d'intellectuel.le.s espagnol.e.s, issu.e.s des mondes de l'université, de la culture, du journalisme et du militantisme. Cette initiative est le fruit du rapprochement entre un groupe de chercheur.e.s en science politique de l'Université Complutense de Madrid - parmi lesquel.le.s Iñigo Errejón, Juan Carlos Monedero et Carolina Bescansa - et les dirigeant.e.s du parti d'extrême-gauche Izquierda Anticapitalista - parmi lesquel.le.s Jaime Pastor et Teresa Rodríguez. Le texte défend la nécessité de " convertir l'indignation sociale " exprimée en 2011 en "majorité électorale et politique " à même de combattre les politiques d'austérité exigées par l'Union Européenne. Le 11 mars suivant, Podemos se constitue juridiquement en tant que parti politique. Mais qualifier Podemos de "parti politique" suffit-il à comprendre ce qui se joue au sein de la formation de Pablo Iglesias? Pourquoi les militant.e.s se méfient-ils.elles de cette appellation? Pourquoi préfèrent-ils.elles le syntagme "parti-mouvement "? Et quelles fonctions remplit cette étiquette? Je tenterai d'apporter des éléments de réponse en m'appuyant sur une analyse du discours des dirigeant.e.s de l'organisation et des observations participantes au sein des cercles de Paris (septembre - décembre 2015) de Poble Sec (Barcelone, septembre-décembre 2016), et d'Arganzuela (Madrid, depuis septembre 2017), ainsi que des observations ponctuelles dans les cercles de Lavapiés (Madrid) et Sant Martí (Barcelone).

\section{Par Manuel \\ Cervera- \\ MARZAL*}

* Post-doctorant à la Casa de Velázquez et chercheur au CESPRA (EHESS). 


\section{- Podemos, davantage qu'un parti politique ?}

En raison de sa fulgurante capacité à faire vaciller le monopole des deux grands partis - PP (Partido popular ou Parti populaire, conservateur) et PSOE (Partido Socialista Obrero Español ou Parti socialiste ouvrier espagnol, social-démocrate) - qui se partageaient la domination du champ politique espagnol depuis trois décennies, on peut se demander si Podemos constitue un parti "comme les autres". S'interroger sur l'éventuelle singularité de Podemos oblige à s'arrêter sur la configuration globale dans laquelle émerge ce parti : configuration profondément marquée par le mouvement dit des Indignés (ou du 15-M) qui, en mai 2011, vit des centaines de milliers d'Espagnol.e.s occuper pendant plusieurs semaines les places publiques de dizaines de grandes villes. Ce cycle contestataire se poursuivit en juillet par une vingtaine de marches citoyennes réunissant plus d'un million de manifestant.e.s. En 2012, d'importantes mobilisations émergent afin de défendre les services publics. Chacune de ces " marées " s'identifie par la couleur de ses drapeaux : vert dans l'éducation, blanc dans la santé, jaune dans les bibliothèques, etc.

Podemos entretient un rapport étroit avec ces mobilisations. Souligner cet aspect ne revient pas à souscrire à l'idée éculée selon laquelle Podemos constituerait " le parti des Indignés " - idée intenable dans la mesure où, premièrement, d'autres partis et organisations sont nés à la suite du 15-M et où, deuxièmement, Podemos procède d'autres sources que le 15-M (les gouvernements socialistes sud-américains, les mobilisations étudiantes et pacifistes des années 2000). Mais cela revient à inscrire Podemos dans la phase ouverte en 2011, c'est-à-dire à être attentif au contexte qui lui donne sens. Cette mise en perspective historique soulève une interrogation : Podemos est-il uniquement un parti politique?

Un faisceau de trois indices suggère que nous aurions tort de réduire Podemos au seul statut de parti politique. Si Podemos est peut-être davantage qu'un parti, c'est d'abord parce qu'avant de se constituer en

\section{Un faisceau de trois indices suggère que nous aurions tort de réduire Podemos au seul statut de parti politique.} tant que tel, le noyau dur des dirigeant.e.s issu.e.s de la Complutense a animé durant plusieurs années une émission télévisée de débats (La Tuerka) qui a progressivement accru son audience jusqu'à devenir l'émission politique la plus regardée d'Espagne. "Si le média ne vient pas à toi, deviens-toi même le média ", affirmaient en 2010 celles et ceux qui fonderont Podemos quatre ans plus tard. C'est par le biais de cet organe audiovisuel que son principal animateur, Pablo Iglesias Turrión, s'est fait connaître auprès du grand public. La figure charismatique d'Iglesias joue un rôle non négligeable dans le succès de Podemos aux élections euro-

1. J. MÜlLER, \#Podemos. Deconstruyendo a Pablo Iglesias, Barcelone, Deusto, 2014, p. 44. péennes de mai 2014 puisque, comme le révèle un sondage publié la veille du scrutin, seuls $7 \%$ des électeurs connaissaient l'existence du parti Podemos... mais $50 \%$ connaissaient la figure de Pablo Iglesias ${ }^{1}$. Le 
lancement de Podemos en tant que parti n'a pas mis fin aux activités de La Tuerka mais a, au contraire, coïncidé avec une diffusion intensifiée (passage d'une fréquence hebdomadaire à une fréquence quotidienne), avec une progression de l'audience et avec le lancement d'une seconde émission politique - Fort Apache - également produite par la société de production (Con Mano Izquierda) appartenant aux dirigeant.e.s de Podemos.

En octobre 2015 fut inauguré dans un quartier populaire de Madrid un immeuble nommé La Morada (L'Habitation), qui constitue le premier jalon d'un réseau de centres sociaux et culturels directement gérés par Podemos. Cet espace de $460 \mathrm{~m}^{2}$ permet aux riverain.e.s de venir boire un verre au comptoir, d'assister à des événements culturels et des expositions photographiques, de s'installer quelques heures dans un espace de coworking ou dans une salle avec accès Wifi, d'acheter des produits dans la boutique

La Morada (L'Habitation), constitue le premier jalon d'un réseau de centres sociaux et culturels directement gérés par Podemos qui s'inspirent explicitement du rôle d'intégration sociale que les partis communistes jouèrent dans l'Europe de l'entre-deux-guerres. officielle de Podemos, d'assister à des débats publics et des projections de films et, enfin, de tenir les réunions des cercles de Podemos ou d'associations locales. Le modèle de la Morada a été dupliqué à Bilbao, Barcelone et dans d'autres municipalités. L'existence de tels centres - qui s'inspirent explicitement du rôle d'intégration sociale que les partis communistes jouèrent dans l'Europe de l'entre-deux-guerres - indique, comme le cas de La Tuerka, que Podemos ne se réduit pas à sa seule dimension partisane.

Un troisième indice invite à se montrer attentif aux multiples facettes du phénomène Podemos. En focalisant le regard sur les cercles, on s'aperçoit que les interactions qui se déploient en leur sein et le travail militant qu'ils déploient diffèrent - parfois fortement - de ce qui se passe traditionnellement dans les organes de base de la plupart des partis politiques. Les réunions des cercles et les activités quotidiennes des militant.e.s ordinaires s'apparentent davantage à ce que font des organisations de mouvement social qu'à ce que produisent les militant.e.s de base des partis institutionnels comme le PP et le PSOE. Concrètement, les membres des cercles Podemos consacrent plus de temps à lutter contre des expulsions locatives, à manifester contre le racisme, à soutenir des grèves dans les services publics et à organiser des actions contre la "touristification " des quartiers populaires de Barcelone et de Madrid qu'à distribuer des tracts, à coller des affiches ou à mener une campagne électorale. Ces observations de terrain sont corroborées par une enquête interne menée à l'automne 2016, qui révèle que $84 \%$ des 778 cercles actifs de Podemos participent à des mouvements sociaux ${ }^{2}$, dont les causes (féminisme,

2. http://www.publico. es/politica/censamillar-circulos-actuarcalle.html (consulté le 15 janvier 2018). 
3. H. Nez, "Podemos, un parti de "non professionnels"? ", in Savoir / Agir, n 32, p. 53-60 ; ainsi que l'article publié par H. Nez dans ce numéro de Mouvements. écologie, migrants, accès à l'éducation, à la santé, à l'énergie) et les modalités (grève, boycott, sit-in, manifestation, occupation, etc.) sont variables.

À ces activités contestataires s'ajoute le fait que les pratiques organisationnelles des cercles - tours de paroles, limitation de la durée des interventions, codes gestuels, modération, féminisation du vocabulaire, etc. - sont en partie héritées des assemblées du 15-Mํ. Enfin, les trajectoires militantes propres aux membres de Podemos témoignent, elles aussi, de la dimension mouvementiste de Podemos. L'intégralité des trente-deux adhérent.e.s, militant.e.s, cadres locaux.ales et dirigeant.e.s nationaux.ales avec qui j'ai mené un entretien semi-directif ont activement participé aux mobilisations du 15-M (contre un taux estimé à $20 \%$ chez la population espagnole d'âge adulte). Ce chiffre concerne un échantillon réduit et mérite d'être corroboré par des données quantitatives plus représentatives. Mais, en dépit de son caractère artisanal, ce décompte comporte une indication précieuse: Podemos recrute en grande partie au sein d'un vivier de militant.e.s protestataires.

\section{- La rue et les urnes}

Podemos peut être qualifié de parti "antisystème " au sens où il ne partage pas les valeurs politiques du système dans lequel il évolue. En effet, Podemos conteste les fondements historiques du régime politique actuel en rappelant à maintes reprises que la Transition démocratique espagnole repose sur "l'impunité des crimes franquistes", sur "l'oubli de la mémoire antifasciste ", et que cette Transition a abouti à la mise en place d'un régime " oligarchique". Podemos conteste également les fondements constitutionnels de ce régime au point de faire de la " réforme de la Constitution de 1978 " l'un des cinq points principaux de son programme électoral. Enfin, Podemos conteste la légitimité du personnel politique espagnol, jugé " corrompu ", " incompétent ", ne représentant que lui-même et "soumis aux intérêts de la Troïka ".

Le caractère antisystème de Podemos se traduit également par la rhétorique que ses membres utilisent pour définir le projet politique du parti : "subvertir le système de l'intérieur ", "jouer le jeu électoral afin de le renverser ", " entrer dans les institutions pour les retourner contre ellesmêmes " et " créer un nouveau parti afin de dépasser les limites des partis traditionnels". Ces formules reviennent souvent dans le discours des militant.e.s. Elles sont le reflet du paradoxe constitutif du militantisme podémiste, à savoir la volonté de changer les règles du champ partisan en s'intégrant à cet univers, la prétention à représenter politiquement un mouvement social fondé sur la critique de la représentation politique.

Le militantisme podémiste est donc pris en tenaille entre une logique d'intégration à l'arène politique institutionnelle (Podemos dans le rôle de 
parti politique) et une logique de contestation (Podemos dans le rôle d'organisation de mouvement social). Ces deux logiques se retrouvent fréquemment dans les partis situés à l'extrême-gauche de l'échiquier politique $^{4}$ et ont déjà été observées au début du $\mathrm{XX}^{\mathrm{e}}$ siècle, quand le mouvement ouvrier s'est institutionnalisé via la création de partis socialistes et communistes, et dans les années 1980, quand le mouvement écologiste a débouché sur la création des partis verts.

Dans le cas de Podemos, la dénonciation radicale du régime politique en vigueur et de ses principaux.ales acteur.trice.s cohabite ainsi avec une participation à la compétition électorale dans le but avoué de conquérir le pouvoir gouvernemental. Ici réside la racine du tiraillement auquel est confronté Podemos. Car, pour accéder au gouvernement, Podemos doit capter un électorat le plus étendu possible, ce qui exige de modérer son offre programmatique, de cultiver une image respectable pour pouvoir prétendre avec crédibilité à l'exercice du pouvoir, et de procéder à certains compromis politiques délivrant des gages de sa capacité à gouverner. Or, toutes ces opérations ne peuvent se faire sans accrocs pour un parti se revendiquant des Indignés, de la contestation sociale, et publiquement opposé aux valeurs du régime au sein duquel il évolue. En s'engageant dans la voie de la normalisation, Podemos prend donc le risque de brouiller son identité contestataire, de perdre en lisibilité auprès de ses électeur.trice.s et de ses sympathisant.e.s, et de s'aliéner la partie de ses militant.e.s la plus attachée à la définition radicale de l'organisation. Inversement, en cultivant son profil subversif, Podemos risque de mettre à mal ses ambitions électorales.

Entre le risque de marginalisation et celui de normalisation, les membres de Podemos sont ainsi tiraillé.e.s. La stratégie de démarcation et celle d'adaptation sont antinomiques. Pourtant, l'une n'exclut pas l'autre puisque la voie des urnes et celle de la rue cohabitent depuis la création du parti. Ces deux stratégies donnent lieu à des divisions internes qui rythment la vie de l'organisation à intervalles réguliers, en particulier lors des congrès nationaux ou locaux.

À l'origine, le principal clivage oppose le groupe de chercheur.e.s en science politique de la Complutense (préalablement fédéré au sein du collectif universitaire Contrapoder créé en 2006, puis de l'émission télévisée La Tuerka lancée en 2010) et le parti d'extrême-gauche Izquierda Anticapitalista (IA, fondé en 2008). Ces deux groupes s'allient en janvier 2014 pour lancer Podemos, mais ils s'affrontent publiquement à des élections internes lors du congrès fondateur des 18 et 19 octobre 2014 puis en juillet 2015 (afin de désigner les candidat.e.s de Podemos aux élections générales de décembre 2015) et lors de la deuxième assemblée citoyenne
4. La question du positionnement de Podemos sur l'échiquier politique traditionnel est complexe puisque 1) certain.e.s dirigeant.e.s nationaux.ales refusent d'affirmer que leur parti est "de gauche " et se présentent comme le "parti du peuple " contre les " partis des élites " (PP, PSOE et Ciudadanos) et 2) une enquête statistique menée par le Consejo Superior de Investigaciones Científicas au lendemain des élections de mai 2014 montre que les électeur.trice.s de Podemos s'autopositionnent au centregauche de l'échiquier politique (en moyenne ils et elles se placent eux.elles-mêmes au niveau de l'indice 3,9 en sachant que 1 = extrême-gauche, $5=$ centre et $10=$ extrême-droite). Cf. J.L. Torreblanca, Asaltar los cielos : Podemos o la política después de la crisis, Madrid, Debate, 2015, p. 41.

\section{Ces deux stratégies donnent lieu à des divisions internes qui rytbment la vie de l'organisation à intervalles réguliers, en particulier lors des congrès nationaux ou locaux.}


5. R. LEFEBVRE, Les primaires socialistes, la fin du parti militant, Paris, Raisons d'Agir, 2011.

de Podemos Madrid, en décembre 2017. À ces différentes occasions, les débats sont tranchés par une procédure de primaires ouvertes auxquelles tout.e Espagnol.e peut voter après s'être inscrit.e gratuitement sur Internet. Dans le cas du Parti socialiste français, Rémi Lefebvre a montré que 1) les primaires accordent un poids prépondérant aux sympathisant.e.s au détriment des militant.e.s, 2) sous couvert de démocratisation les primaires tendent à renforcer le pouvoir des dirigeant.e.s nationaux.ales et 3) que les primaires constituent une arme dans les luttes internes aux partis 5 . Malgré les différences de contexte, ces points semblent valables dans le cas de Podemos. Un élément en témoigne : la procédure des primaires a été imposée par l'équipe de la Complutense contre la volonté d'Izquierda Anticapitalista (IA) qui voulait réserver le vote aux militant.e.s, or la plupart des sondages montrent que les sympathisant.e.s votent davantage pour l'équipe d'Iglesias que ne le

En dépit de leur opposition, les rivaux s'accordent donc sur l'idée que Podemos doit tenir les deux bouts à la fois : efficacité et radicalité, élections et contestation, urnes et rues. font les militant.e.s. Pour les dirigeant.e.s de la Complutense, les primaires ouvertes permettent de court-circuiter les cercles locaux dont une partie non négligeable est plus sensible à la ligne d'IA qu'à celle d'Iglesias. Cependant, les tendances de Podemos les plus impliquées dans les mouvements sociaux ne se réduisent pas à une minorité passive servant de "caution " contestataire à Iglesias puisqu'elles dirigent le parti dans des régions importantes comme l'Aragon (jusqu'à ce que le secrétaire régional, Pablo Echenique, rejoigne en 2016 les positions d'Iglesias) et l'Andalousie et qu'elles sont fortement implantées dans des villes comme Madrid, Malaga, Cadix et Pampelune.

Lors d'une conférence prononcée en janvier 2015 par Carolina Bescansa, celle qui était à l'époque numéro trois de l'équipe de la Complutense affirma, à l'encontre de ses concurrents d'IA, qu'il existait " un Podemos pour gagner, gouverner et impulser le changement politique

6. http://www. europapress.es/ nacional/noticiapodemos-apoya-alegredirigir-partido-madridfrente-oponentes-soncandidatura-protestar $-20150119173200 . \mathrm{html}$ (consulté le 15 janvier 2018) dans notre pays, et un autre Podemos pour protester "6. Après avoir apporté son soutien intégral au "Podemos pour gagner ", Bescansa ajouta néanmoins que "les deux Podemos sont légitimes dans l'organisation " et que le second, " plus orienté vers le domaine de la protestation ", a " beaucoup à apporter " à l'organisation. Sociologue de profession, Bescansa s'exprimait ici en tant que dirigeante politique. Quand Bescansa énonça cette opposition " Podemos pour gagner / Podemos pour protester ", elle était prise dans une logique de lutte interne devant aboutir à l'élection du secrétaire général de Podemos Madrid (le candidat Luis Alegre était soutenu par Iglesias et Bescansa, tandis que le candidat Miguel Urbán était soutenu par IA). À cette attaque, Miguel Urbán répondit le lendemain : "C'est en protestant que nous avons gagné la mairie de Madrid " et " je me revendique du Podemos de la protestation qui est celui qui nous permet- 
tra de gagner " ${ }^{7}$. En dépit de leur opposition, les rivaux s'accordent donc sur l'idée que Podemos doit tenir les deux bouts à la fois : efficacité et radicalité, élections et contestation, urnes et rues.

Décisif au cours des deux premières années d'existence de Podemos (2014-2015), le clivage entre l'équipe de la Complutense et les membres d'Izquierda Anticapitalista est ensuite parfois passé au second plan en raison d'une scission au sein du groupe de la Complutense, entre " pablistes " et " errejonistes ", scission atteignant son acmé lors de la seconde assemblée citoyenne de Podemos, en février 2017. Des alliances locales et ponctuelles entre pablistes et anticapitalistes, ainsi que leur volonté commune de rester ferme dans les négociations gouvernementales de 2016 avec le PSOE, ont également complexifié le clivage initial. Il n'en reste pas moins que les militant.e.s d'IA conservent une position spécifique au sein du parti, en raison de leur regroupement dans certains cercles et de leur forte propension à s'engager dans les mouvements sociaux.

Dans la mesure où Podemos s'inscrit à la fois dans et contre le jeu institutionnel, où il agit en tant que parti politique (visant la conquête du pouvoir) et en tant que mouvement social (cherchant à contester le pouvoir), nous sommes autorisés à qualifier cette organisation militante de parti-mouvement. Un regard chronologique invite cependant à ne pas figer ce constat. Alors qu'en 2014 Podemos se présente comme un mouvement et refuse l'étiquette de parti, que ses cercles connaissent une rapide extension (jusqu'à 1500 cercles actifs sur le territoire) et adoptent spontanément un modèle auto-organisationnel, cette effervescence retombe lors de la série de campagnes électorales (municipales, régionales, générales) de 2015. Le nombre de cercles diminue, l'informalité est mise à mal par une procédure via laquelle les instances officielles "valident " l'existence des cercles de base, ainsi que par une perte de pouvoir de ces mêmes cercles. Cette normalisation de Podemos s'enraye néanmoins en 2016 avec la création de nouveaux cercles et un regain d'enthousiasme chez les militant.e.s. Au printemps 2016, la nomination de Pablo Echenique (réputé proche de la base du parti) au poste de secrétaire d'organisation est accueillie positivement par la majorité des militant.e.s. Le 31 octobre suivant, l'investiture de Mariano Rajoy comme président du gouvernement met un terme aux aspirations gouvernementales de Podemos. Le parti, cantonné à un rôle d'opposition, redécouvre alors la fibre mouvementiste qui l'animait à sa fondation.

Mais surgit ici une difficulté : si la notion de "parti-mouvement " est une catégorie scientifique à disposition du ou de la sociologue, elle est
7. http://www. elmundo.es/madrid/2 015/01/20/54be51c1ca 4741087a8b4575.html (consulté le 15 janvier 2018). 
aussi une catégorie pratique explicitement revendiquée par les acteurs et mobilisée dans la compétition interpartisane.

\section{- Les usages stratégiques de l'expression " parti-mouvement »}

"C'est quand la dernière fois que vous avez voté avec espoir?" Le slogan de campagne de Podemos aux élections européennes de 2014 résume bien la stratégie de ce parti : susciter de l'enthousiasme dans un pays

\section{Le recours à l'expression} "parti-mouvement " constitue une ressource symbolique précieuse, employée dès la genèse de Podemos et permettant de se distinguer des autres partis. frappé par la crise sociale et économique - entre 2007 et 2012 le chômage est passé de $7 \%$ à $25 \%$ et la dette publique de $36 \%$ à $100 \%$ du PIB - où le taux d'abstention s'élève de façon structurelle depuis une décennie. Ce travail de réenchantement de la vie politique passe par la promotion de figures méconnues du grand public et par le recours à un

lexique politique renouvelé. Sur ce dernier aspect, certains changements rhétoriques ont suscité de nombreux commentaires : l'abandon des références à " la classe ouvrière " voire même à la "gauche " au profit d'un appel au " peuple " (contre " la caste "), aux " gens " (contre " les élites "), aux " citoyens" (contre "l'oligarchie ") et à "la patrie " (contre les politi-

8. Peut-être serait-il plus juste de parler de " démarxisation" ou de "désouvriérisation " que d'une désidéologisation, car changer d'idéologie n'est pas abandonner toute idéologie. Je pointe ici l'abandon progressif des références au marxisme et à la classe ouvrière dans le discours des dirigeant.e.s (mais tout autant des militant.e.s) de Podemos.

9. O. KirchmeIER, "The Transformation of the Western European Party Systems ", in $L a$ Palombara, Weiner (eds.), Political Parties and Political Development, Princeton University Press, Princeton, 1966. ciens et banquiers qui " trahissent " la patrie). Cette désidéologisation ${ }^{8}$ de Podemos - assumée par une partie des dirigeant.e.s, proches d'Iñigo Errejón - rappelle à plusieurs égards la façon dont au cours des années 1960 certains partis "attrape-tout " ont dilué leur base idéologique afin d'élargir leur base électorale?.

Un autre élément de langage, passé inaperçu, me semble au moins aussi décisif que ceux mentionnés ci-dessus. Il s'agit du recours à l'expression " parti-mouvement ". Cette expression constitue une ressource symbolique précieuse, employée dès la genèse de Podemos et permettant de se distinguer des autres partis. Alors que partis politiques et mouvements sociaux sont généralement conçus comme des entités distinctes voire incompatibles, leur juxtaposition permet de souligner la nature hybride de Podemos, de montrer que cette organisation se situe au carrefour de la rue et des urnes, de la contestation et de l'institution. Cette mise en scène discursive du tiraillement produit l'image d'un parti " pluraliste ", " ouvert " et "incertain " qui, par là même, se distingue des partis " monolithiques ", " dogmatiques" et "pétris de certitudes " tels que le PP et le PSOE.

Aux élections générales de juin 2016, Podemos s'allie avec son rival d'ascendance communiste, Izquierda Unida (IU), pour former la liste Unidos Podemos. Auparavant, c'est-à-dire durant ses deux premières années de vie (2014-2015), Podemos prenait ses distances avec IU. Tandis que de nombreux journalistes espagnol.e.s faisaient régulièrement remarquer que le programme politique et économique de Podemos était à peu près identique à celui de son concurrent Izquierda Unida, cette proximité était 
démentie par les représentant.e.s de Podemos, qui se démarquaient d'IU en attirant l'attention du public sur la forme organisationnelle novatrice de Podemos. C'est généralement ici qu'intervenait l'expression " parti-mouvement ", grâce à laquelle les podémistes s'autorisaient à affirmer, comme le fit Pablo Iglesias dans un petit livre à succès : "Si Podemos s'est constitué en parti, c'est pour des raisons d'impératif légal, pour pouvoir participer aux élections, même si, fondamentalement, nous restons un mouvement, un " mouvement de mouvements"

\section{"Si Podemos s'est constitué en parti, c'est pour des raisons d'impératif légal, pour pouvoir participer aux élections, même si, fondamentalement, nous restons un mouvement».}

comme nous aimons à le dire. Les

cercles Podemos, ces assemblées de citoyens qui ont surgi et continuent de surgir spontanément à travers le pays dans les villages, les quartiers, les villes, sont des assemblées de base, informelles, sans dirigeants ni cotisations, incluant de vingt à trois cents personnes. Ce sont des espaces pour en finir avec la peur, la fragmentation, la résignation. Leur rôle a été essentiel dans notre succès lors des élections européennes du 25 mai 2014. Ils ont constitué cette articulation entre le peuple et le mouvementparti qu'est Podemos. Aujourd'hui, ils sont plus d'un millier, répartis sur tout le territoire espagnol, mais aussi hors d'Espagne " $"$.

L'alliance de 2016 avec Izquierda Unida, reconduite lors des élections suivantes, constitue un tournant stratégique majeur dans la (courte) histoire de Podemos. Ce tournant stratégique s'accompagne d'un tournant sémantique dans la mesure où, progressivement, l'expression " parti-mouvement" se fait plus rare (sans pour autant disparaître) dans la bouche et sous la plume des dirigeant.e.s de Podemos. Cette expression reste en revanche fréquemment mobilisée par les membres des cercles.

Quelle fonction cette expression remplit-elle ? Notons déjà qu'elle permet aux membres de Podemos d'éviter le label " parti ", fortement déprécié dans l'imaginaire espagnol puisqu'il évoque des organisations " archaïques ", " corrompues " et " coupées des gens ordinaires ". Le mot " parti " fait naître spontanément à l'esprit les deux entités qui se partagent en alternance le pouvoir depuis la transition démocratique : le PP et le PSOE. Les membres de Podemos assimilent le PPSOE (contraction de PP et PSOE, à la manière de l'" UMPS " vilipendé par le Front national) à la "vieille politique ". Accoler le terme " mouvement "à celui de " parti " permet d'éviter le stigmate partisan, de présenter Podemos comme " le parti des Indignés " et de s'attribuer ainsi le capital de sympathie dont jouissait et jouit encore ce mouvement, puisqu'en 2014 plus de $70 \%$ des Espagnol.e.s disent en " partager le message et les revendications " ${ }^{11}$. L'expression "parti-mouvement " constitue ainsi une ressource symbolique ayant vocation à montrer à l'électorat que Podemos n'est pas un parti comme les autres et représente la seule force capable de "convertir l'indignation sociale en changement politique ".
10. Pablo Iglesias, cité dans A. Dominguez, L. Gimenez (dir.), Podemos, sûr que nous pouvons!, Montpellier, Indigènes, 2015, p. 91.

11. J. Rivero, Podemos. Objetivo: Asaltar los cielos, Madrid, Planeta, 2015, p. 101-114; et http://metroscopia.org/ el-apoyo-ciudadano-al$15 \mathrm{~m}$-tres-anos-despues/ (consulté le 15 janvier 2018). 
12. https://gaceta es/espana/dimitenmas-cargos-podemosincluida-la-diputadaquito-las-banderas -20171107-1112/ (consulté le 15 janvier 2018).
Au-delà de la ressource que représente la notion de "parti-mouvement ", les membres de Podemos peuvent être amené.e.s à mettre en scène le tiraillement de leur organisation afin de promouvoir ce produit politique. Les militant.e.s peuvent effectivement capitaliser sur leurs dilemmes, les mettre en récit afin de donner l'image d'une organisation saisie par le doute et l'autocritique, qui se démarque ainsi des concurrents décriés pour leur incapacité à remettre en question leurs vieilles habitudes. Afin de séduire et de recruter un stock de déçu·e.s du militantisme partisan en attente d'une nouvelle organisation plus ouverte que les autres, les représentant.e.s de Podemos ont un certain intérêt à rendre publiques les luttes intestines qui traversent leur organisation. Alors que la sociologie de l'action collective met généralement l'accent sur le travail d'unification opéré par les leaders et sur la construction d'une image publique homogène, le cas de Podemos illustre à l'inverse combien un groupe peut tirer profit de la mise en scène de ses divisions. Mais, si elles peuvent par moments se révéler profitables politiquement, ces divisions risquent toujours de détériorer l'image du parti, d'affecter ses propres membres, voire de conduire à des départs, comme ce fut le cas de militant.e.s catalans en novembre 2017, lorsque Podemos se divisa sur la question de l'indépendance catalane ${ }^{12}$.

L'usage stratégique du label "parti-mouvement " ne signifie pas que Podemos procède à une dévalorisation intégrale du label " parti ". Il semblerait plutôt que les représentant.e.s de Podemos soient pris.es dans un dilemme quant à l'utilisation de ce label : d'un côté, il faut réhabiliter le qualificatif de "parti " pour se démarquer des militant.e.s contestataires hostiles à toute participation aux élections et donc "prisonnier.ère.s " d'une posture " inoffensive ", " impuissante ", " puriste " et " anarchiste "; d'un autre côté, les dirigeant.e.s de Podemos rechignent à se présenter comme un " parti " car ce mot leur sert de repoussoir qui permet de faire d'une pierre deux coups en s'attaquant simultanément au PP et au PSOE, mis dans le même sac " partisan ". Pour les militant.e.s de Podemos, le mot " parti " est donc un terme à double usage, ou à usage flottant, dans la mesure où il sert à la fois à défendre leur pratique - contrairement aux contestataires anarchistes, les membres de Podemos n'ont pas peur de mettre les mains dans le cambouis, d'entrer dans les institutions et de prendre des responsabilités - et à

stigmatiser les pratiques de leurs concurrents - complices du système, englués dans des habitudes mortifères.

À cet égard, l'usage stratégique des dilemmes est une arme à double tranchant, qui peut se retourner contre ceux et celles qui la mettent 
en œuvre. En effet, la mise en scène du tiraillement permet de donner l'image d'un parti pluraliste plus attractif que ses concurrents mais, dans le même temps, les concurrents apprennent à se saisir des désaccords publics entre les différentes composantes de Podemos pour dénoncer le manque de crédibilité de ce parti et pour souligner que les électeur.trice.s ne peuvent pas sérieusement confier les rênes du gouvernement à une organisation dont les membres sont incapables de se mettre d'accord sur une base commune.

Pour conclure, précisons que l'expression " parti-mouvement " ne sert pas uniquement à se démarquer des partis concurrents. Elle est aussi mobilisée dans les luttes intestines qui traversent Podemos. Si Pablo Iglesias et Iñigo Errejón ne convoquent presque plus ce syntagme depuis 2016, il reste en revanche utilisé par les dirigeant.e.s d'Anticapitalistas. Dans un entretien accordé au journal El Diario en janvier 2018, à l'occasion du quatrième anniversaire de Podemos, Miguel Urbán dresse le bilan suivant : "Il nous faut impérativement revenir à l'élan de débordement populaire qui fut à l'origine de Podemos et qui surprit ses propres fondateurs, revenir aux luttes concrètes des gens ordinaires, nous avons besoin d'un parti-mouvement, nous avons besoin de réinventer Podemos afin qu'il ne se convertisse pas en un énième parti traditionnel mais en un outil des classes populaires grâce auquel elles puissent changer leur vie, et changer le monde "13. Deux ans et demi plus tôt, dans un communiqué officiel parti le 31 octobre 2015 à propos des élections générales, Anticapitalistas critiquait déjà la direction iglésiste du parti, à laquelle était reprochée l'absence de volonté " de s'implanter dans la classe travailleuse afin d'y construire un parti-mouvement qui se nourrisse et qui nourrisse le conflit social "14. Ces recours répétés à l'expression "parti-mouvement " permettent au courant le plus contestataire de Podemos de mettre en garde les fractions rivales, qui seraient tentées par une définition exclusivement institutionnelle et électorale de cet outil collectif.
13. http://www. eldiario.es/politica/ anos-PodemosDijeron-plazaspuede_0_729327145. html (consulté le 15 janvier 2018 ; je traduis).

14. http://www. anticapitalistas. org/comunicados/ declaracion-deanticapitalistas-niregeneracion-niresignacion-si-podemos/ (consulté 15 janvier 2018 ; je traduis). 